\title{
Absorption of an electron by a dielectric wall
}

\author{
F. X. Bronold and H. Fehske \\ Institut für Physik, Ernst-Moritz-Arndt-Universität Greifswald, 17489 Greifswald, Germany
}

(Dated: August 20, 2021)

\begin{abstract}
We introduce a method for calculating the probability with which a low-energy electron hitting the wall of a bounded plasma gets stuck in it and apply the method to a dielectric wall with positive electron affinity smaller than the bandgap using $\mathrm{MgO}$ as an example. In accordance with electron beam scattering data we obtain energy-dependent sticking probabilities significantly less than unity and question thereby for electrons the perfect absorber assumption used in plasma modeling.
\end{abstract}

PACS numbers: 68.49.Jk, 79.20.Hx, 52.40.Hf

The interaction of electrons with surfaces plays a key role in applied science. Various methods of surface analysis [1-3] are based on it as well as a number of materials processing techniques [4]. In these applications the electron energy is above $100 \mathrm{eV}$ and backscattering and secondary electron emission, the physical processes involved, are sufficiently well understood [5-12]. The situation is different for electron-surface interaction at energies below $100 \mathrm{eV}$, as it occurs in dielectric barrier discharges 13 15], dusty plasmas [16 20], Hall thrusters [21, 22], and electric probe measurements [23]. Much less is quantitatively known about it, especially at very low energies, below $10 \mathrm{eV}$. For instance, the probability with which a low-energy electron gets stuck in the wall after hitting it from the plasma is unknown. In the modeling of bounded plasmas 24 28 it is assumed to be close to unity, implying for electrons the wall is a perfect absorber [29], irrespective of the material. Since electron absorption and extraction (by charge-transferring heavy particle collisions) control the wall potential, and hence the plasma sheath, which in turn affects the bulk plasma, the electron sticking probability is a crucial parameter. That its magnitude matters and should be known precisely has been recognized most clearly by Mendis [20] in connection with grain charging in dusty plasmas but the theoretical work [30, 31] he refers to is based on classical mechanics not applicable to electrons.

In this work we apply quantum mechanics to calculate the probability with which a low-energy electron is absorbed by a surface. We couch the presentation in a particular application: The calculation of the electron sticking probability at room temperature for a dielectric wall 32] with positive electron affinity $\chi$ smaller than the bandgap $E_{g}$. But the approach is general and can be also applied to other cases. It utilizes two facts noticed by Cazaux [1]: (i) low-energy electrons do not see the strongly varying short-range potentials of the surface's ion cores but a slowly varying surface potential and (ii) they penetrate deeply compared to the lattice constant into the surface. The scattering pushing the electron back to the plasma occurs thus in the bulk of the wall suggesting the probability for the electron to get absorbed by it to be the probability for transmission through the wall's surface potential times the probability to stay inside the wall despite of internal backscattering.

The variation of the potential across a floating dielectric wall can be calculated self-consistently 33]. For $\chi>0$ it gives rise to an energy barrier for electrons whose height on the plasma side is the Coulomb energy $U_{\mathrm{w}}$ an electron has to overcome to reach the wall. On the solid side the height is $\chi$ since for $\chi>0$ electrons entering the wall and making up its charge are surplus electrons occupying the wall's conduction band [33]. For the sticking probability it is the kinetic energy of the approaching electron in the vicinity of the wall which matters. It suffices therefore to model the wall by a three-dimensional potential step with height $\chi$ and electron mass mismatch $\bar{m}_{e}=m_{e}^{*} / m_{e}<1$, where $m_{e}^{*}$ is the effective electron mass in the conduction band of the wall and $m_{e}$ is the electron mass, as illustrated in Fig. 17. More refined treatments are possible. The shape of the step however is largely irrelevant for the energies we consider. For $\mathrm{Al}_{2} \mathrm{O}_{3}$, for instance, the attenuation length for an electron with a few $\mathrm{eV}$ is around $200 \AA$ [34] and thus much larger than the lattice spacing setting the scale on which the step varies.

Assuming as in Fig. 1h the wall (plasma) to occupy the $z<0(z>0)$ half space, measuring energy in Rydbergs from the bottom of the conduction band, that is, setting $E_{\mathrm{cb}}=U_{\mathrm{w}}-\chi \equiv 0$, length in Bohr radii, and mass in electron masses, the transmission probability for a plasma electron hitting the wall, and having thus kinetic energy $E-\chi>0$, is given by 35$]$

$$
\mathcal{T}(E, \xi)=\frac{4 \bar{m}_{e} k p}{\left(\bar{m}_{e} k+p\right)^{2}}
$$

with $k=\sqrt{E-\chi} \xi$ and $p=\sqrt{\bar{m}_{e} E} \eta$ the $z$-components of the electron momenta outside and inside the wall. In (11) the signs of $k$ and $p$ are always the same. We can thus define the direction cosines $\xi$ and $\eta$ referenced, respectively, to the electron momenta outside and inside the wall, by their absolute values: $\xi=|\cos \beta|$ and $\eta=|\cos \theta|$ (see Fig. 1 $\mathrm{b}$ for the definition of $\beta, \theta$, and $E)$. This choice is dictated by the modeling of internal backscattering presented later. Since the potential varies only perpendicularly to the interface the lateral momentum $\vec{K}$ is conserved. Together with energy conservation, 


$$
\begin{gathered}
E=\chi+k^{2}+\vec{K}^{2}=\left(p^{2}+\vec{K}^{2}\right) / \bar{m}_{e}, \text { this yields } \\
1-\eta^{2}=\frac{E-\chi}{\bar{m}_{e} E}\left(1-\xi^{2}\right)
\end{gathered}
$$

connecting $\eta$ and $\xi$. From (2) follows that an electron from the plasma approaching the wall with kinetic energy $E-\chi=\vec{K}^{2}+k^{2}>0$ enters it only when

$$
\xi>\xi_{c}= \begin{cases}0 & \text { for } \chi<E<E_{0} \\ \sqrt{1-\frac{\bar{m}_{e} E}{E-\chi}} & \text { for } E>E_{0}\end{cases}
$$

with $E_{0}=\chi /\left(1-\bar{m}_{e}\right)$. For $\xi<\xi_{c}$ the electron is in an evanescent wave with $p^{2}<0$, once it is inside the wall, and is thus totally reflected [36]. This fact, unnoticed so far in connection with dielectric walls, has strong implications for the sticking probability. In addition, Eq. (2) may force an electron entering the wall from the plasma to instantaneously acquire inside the wall a perpendicular kinetic energy $p^{2} / \bar{m}_{e}<\chi$. For $\bar{m}_{e}<1$, applicable to $\mathrm{MgO}$, the material we use for illustration because of the availability of data from electron beam scattering experiments [37], this happens when $\xi<\sqrt{1-\bar{m}_{e}}$. If such an electron cannot gain energy by inelastic scattering, as it is the case for dielectric walls at room temperature, it will have no chance to come back to the plasma since it will not be able to overcome the potential step $\chi$.

The transmission probability captures only the ballistic aspects of electron absorption by the wall. Once the electron is inside the wall it is subject to elastic and inelastic internal scattering, which may push the electron back to the interface and, after successfully traversing the surface potential in the reverse direction, back to the plasma (see Fig. 17 for an illustration). To take this possibility into account we define the sticking probability $S(E, \xi)$ as the probability of an electron hitting the wall from the plasma with energy $E$ and direction cosine $\xi$ not to return to it after entering the wall and suffering backscattering inside it. Hence,

$$
S(E, \xi)=\mathcal{T}(E, \xi)[1-\mathcal{E}(E, \xi)]
$$

with the conditional probability

$$
\mathcal{E}(E, \xi)=\frac{\int_{\eta_{\min }}^{1} d \eta^{\prime} \int_{E_{\min }^{\prime}}^{E} d E^{\prime} \rho\left(E^{\prime}\right) Q\left(E \eta(\xi) \mid E^{\prime} \eta^{\prime}\right) \mathcal{T}\left(E^{\prime}, \xi\left(\eta^{\prime}\right)\right)}{\int_{0}^{1} d \eta^{\prime} \int_{0}^{E} d E^{\prime} \rho\left(E^{\prime}\right) Q\left(E \eta(\xi) \mid E^{\prime} \eta^{\prime}\right)}
$$

for the electron to escape from the wall after at least one backscattering event. The lower integration limits in the numerator, $\eta_{\min }=\sqrt{\chi / E}$ and $E_{\min }^{\prime}=\chi / \eta^{\prime 2}$, ensure that only events are counted for which the perpendicular post-collision energy $p^{\prime 2} / \bar{m}_{e}>\chi, \rho(E)=\sqrt{\bar{m}_{e}^{3} E} / 2(2 \pi)^{3}$ is the conduction band density of states, and $Q\left(E \eta \mid E^{\prime} \eta^{\prime}\right)$ is proportional to the probability for an electron, penetrating the wall in a state with energy $E$ and direction

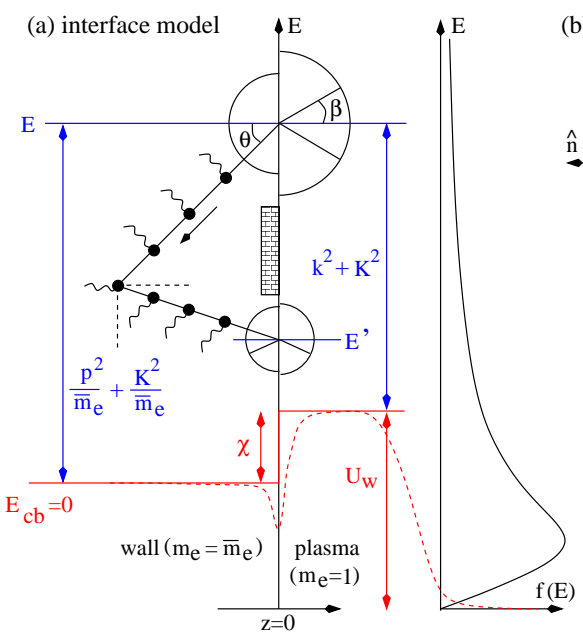

(b) calculational approach

FIG. 1. (color online) (a) Potential energy step of height $\chi$ modeling the wall and a scattering trajectory bringing an electron entering the wall back to the plasma. Half circles are the moduli of the electron momenta outside and inside the wall at the entrance $(E)$ and exit $\left(E^{\prime}\right)$ point connected by phonon emission events. Also shown is the Coulomb barrier $U_{\mathrm{w}}$ the electron has to overcome to reach the wall, the energy distribution $f(E)$ it may have, and a realistic surface potential (red dashed curve). The tilt of the potential step due to the wall charge responsible for $U_{\mathrm{w}}$ is not shown. (b) Illustration of Eqs. (4) - (5) defining $S(E, \xi)$. The potential step leads to a transmission probability $\mathcal{T}$ while the emission of phonons to a conditional backscattering probability $\mathcal{E}$ proportional to a quantity $Q$, obtained from the invariant embedding approach, that is, by adding an infinitesimally thin layer to the wall and requiring $Q$ to be invariant against it [12]. Notice the definition of the angles and energies characterizing the electron. Inside (outside) the wall the angles, measured with respect to the surface normal $\hat{n}$, are denoted $\theta(\beta)$ and $\theta^{\prime}\left(\beta^{\prime}\right)$.

cosine $\eta$, to backscatter after an arbitrary number of internal scattering events towards the interface in a state with energy $E^{\prime}$ and direction cosine $\eta^{\prime}$ (see Fig. 10). Since $Q\left(E \eta \mid E^{\prime} \eta^{\prime}\right)$ describes backscattering, $0<\theta \leq \pi / 2$ and $\pi / 2<\theta^{\prime} \leq \pi$, implying $0 \leq \eta, \eta^{\prime}<1$ as $\eta^{\prime}=\left|\cos \theta^{\prime}\right|$. The energy integrals in (5) anticipate that for a dielectric wall at room temperature the electron cannot gain energy and $\eta(\xi)$ and its inverse $\xi(\eta)$ are defined by Eq. (2).

To calculate $Q\left(E \eta \mid E^{\prime} \eta^{\prime}\right)$ we use the invariant embedding approach, originally applied to the electron backscattering problem by Dashen [12] and revitalized recently by Vicanek [8] and Glazov and coworkers [5, 38]. It is illustrated in Fig. 1b. Summing up the four paths (1)-(4), requiring $Q$ to be invariant against the change it induces, and taking into account that $Q$ does not depend on the azimuths of the electron's initial and final propagation direction measured with respect to the surface normal $\hat{n}$ yields a nonlinear integral equation for $Q\left(E \eta \mid E^{\prime} \eta^{\prime}\right)[12,38$. Since at low energy most scattering processes in a solid are forwardly peaked [39] we linearize the equation by an expansion of $Q\left(E \eta \mid E^{\prime} \eta^{\prime}\right)$ in the 
number of the rare backscattering events. For dielectric surfaces at room temperature and $0<\chi<E_{g}$ scattering at low energy arises mainly from the emission of optical phonons with energy $\omega$. Since the electron cannot gain energy by scattering it can loose at most the energy it initially had when entering the wall. Expanding $Q\left(E \eta \mid E^{\prime} \eta^{\prime}\right)$ also in the number of forward scattering events yields thus a double series which terminates after a finite number of terms. From the golden rule transition rate per time [40] and the material parameters of $\mathrm{MgO}$ [41, 42] follows that backward scattering due to emission of an optical phonon is two orders of magnitude less likely than forward scattering. Writing

$$
Q\left(E \eta \mid E^{\prime} \eta^{\prime}\right)=\sum_{n=1}^{\infty} \sum_{m=0}^{\infty} Q_{m}^{n}\left(E ; \eta \mid \eta^{\prime}\right) \delta\left(E-E^{\prime}-\omega_{m}^{n}\right)
$$

with $\omega_{m}^{n}=(n+m) \omega$ we can thus truncate the summation already after a single backward scattering event, that is, after $n=1$ leaving $M_{\text {tot }}=\lfloor E / \omega\rfloor-1$ forward scattering events at most. Introducing kernels

$$
\begin{aligned}
K^{ \pm}\left(E \eta \mid E^{\prime} \eta^{\prime}\right) & =\frac{1}{2 \rho(E)}\left[\left(E+E^{\prime} \mp 2 \sqrt{E E^{\prime}} \eta \eta^{\prime}\right)^{2}\right. \\
& \left.-4 E E^{\prime}\left(1-\eta^{2}\right)\left(1-\eta^{\prime 2}\right)\right]^{-1 / 2}
\end{aligned}
$$

for forward $(+)$ and backward $(-)$ scattering and a function $\Pi(E)=\operatorname{arcosh}(\sqrt{E / \omega}) / E$, all three arise in the course of the calculation from the transition rate per length, the input driving the integral equation for $Q$ [38] most directly obtained by dividing the transition rate per time by the electron velocity prior the emission event, the expansion coefficients satisfy

$$
\begin{aligned}
Q_{m}^{1}\left(E ; \eta \mid \eta^{\prime}\right) & =F_{m}\left(E ; \eta \mid \eta^{\prime}\right) Q_{m-1}^{1}\left(E-\omega ; \eta \mid \eta^{\prime}\right) \\
& +Q_{m-1}^{1}\left(E ; \eta \mid \eta^{\prime}\right) G_{m}\left(E-m \omega ; \eta \mid \eta^{\prime}\right)
\end{aligned}
$$

with $m=1, \ldots, M_{\text {tot }}$, and

$$
\begin{aligned}
F_{m}\left(E ; \eta \mid \eta^{\prime}\right) & =\frac{K^{+}(E \mid E-\omega ; \eta) \eta^{\prime} \rho(E-\omega)}{\eta^{\prime} \Pi(E)+\eta \Pi(E-(m+1) \omega)}, \\
G_{m}\left(E ; \eta \mid \eta^{\prime}\right) & =\frac{\eta \rho(E) K^{+}\left(E \mid E-\omega ; \eta^{\prime}\right)}{\eta^{\prime} \Pi(E+m \omega)+\eta \Pi(E-\omega)} .
\end{aligned}
$$

The initialization of the recursion (8) is given by

$$
Q_{0}^{1}\left(E ; \eta \mid \eta^{\prime}\right)=\frac{\eta^{\prime} K^{-}\left(E \eta \mid E-\omega \eta^{\prime}\right)}{\eta^{\prime} \Pi(E)+\eta \Pi(E-\omega)} .
$$

Forward scattering is encoded into the function $K^{+}\left(E \mid E^{\prime} ; \eta\right)=\int_{0}^{1} d \bar{\eta} K^{+}\left(E \eta \mid E^{\prime} \bar{\eta}\right)=\int_{0}^{1} d \bar{\eta} K^{+}\left(E \bar{\eta} \mid E^{\prime} \eta\right)$, where the second identity utilizes the symmetry of $K^{+}\left(E \eta \mid E^{\prime} \eta^{\prime}\right)$ with respect to interchanging $\eta$ and $\eta^{\prime}$. It can be obtained analytically and enters the formalism because $K^{+}\left(E \eta \mid E^{\prime} \eta^{\prime}\right)$ is strongly peaked for $\eta=\eta^{\prime}$, as can be seen from Eq. (7), and noticing that $K^{+}\left(E \eta \mid E^{\prime} \eta^{\prime}\right)$ enters always with $E^{\prime}=E-\omega$. Integrals over the direction
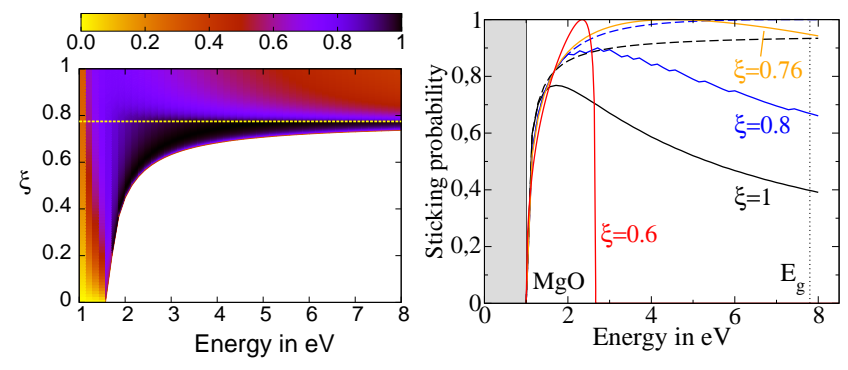

FIG. 2. (color online) Electron sticking probability for $\mathrm{MgO}$ obtained from Eq. (4). On the left is shown $S(E, \xi)$ for all direction cosines $\xi$ and energies $E$ up to the bandgap $E_{g}$. Total reflection occurs in the white region. Below the yellow dotted line inelastic backscattering has no effect on $S(E, \xi)$. On the right $S(E, \xi)$ (solid curves) and $\mathcal{T}(E, \xi)$ (dashed curves) are plotted as a function of $E$ for representative $\xi$. The material parameters are $\bar{m}_{e}=0.4, \chi=1 \mathrm{eV}, E_{g}=7.8 \mathrm{eV}$ [41], and $\omega=0.1 \mathrm{eV}$ [42]. The value for $\bar{m}_{e}$ is strictly applicable only at the band edge. Away from it $\bar{m}_{e}$ depends on momentum. From the bandstructure [4] we expect our results reliable up to $5 \mathrm{eV}$ enough for a comparison with experiment (see Fig. 3).

cosine containing $K^{+}\left(E \eta \mid E^{\prime} \eta^{\prime}\right)$ can thus be handled by a saddle-point approximation. Physically this means the directional change due to forward scattering is negligible. Only backward scattering changes the direction (see Fig. (1a). We thus end up with a model similar in spirit to the Oswald, Kasper and Gaukler model [43] for backscattering of electrons from surfaces due to multiple elastic scattering. The strong forward scattering comes from the matrix element for the interaction of an electron with an optical phonon which is at low electron density inversely proportional to the momentum transfer squared [40].

Substituting expansion (6) for $Q\left(E \eta \mid E^{\prime} \eta^{\prime}\right)$ in (5) sets $E^{\prime}$ to $E_{m}^{1}=E-\omega_{m}^{1}$ and replaces the energy integrals by sums over $m$ running in the numerator up to $m=$ $M_{\text {open }}=\left\lfloor\left(E \eta^{2}-\chi\right) /\left(\eta^{2} \omega\right)\right\rfloor-1$ and in the denumerator up to $m=M_{\text {tot }}$. Inserted into Eq. (4) it finally gives the electron sticking probability $S(E, \xi)$ at room temperature for a dielectric wall with $0<\chi<E_{g}$ and a clean, lateral momentum conserving interface.

In Fig. 2] we present the results for $\mathrm{MgO}$ obtained from Eq. (4). Material parameters are given in the caption. The left panel shows the angle-resolved sticking probability $S(E, \xi)$ over the whole range of direction cosines and energies up to the bandgap, above which electron-hole pair generation across the bandgap has to be taken into account as an additional, Coulomb-driven backscattering process. It can be treated in the same spirit, in particular the truncation after one backscattering event will still be possible, but the recursion will then contain energy integrals. The white area in the plot for $S(E, \xi)$ indicates the region in the $(E, \xi)$-plane where total reflection occurs. It is large because $\bar{m}_{e}$ is for $\mathrm{MgO}$ significantly smaller than unity. Below the dotted yellow line, $\xi=\sqrt{1-\bar{m}_{e}}$, inelastic backscattering due to emission of optical phonons 
is irrelevant for sticking because the perpendicular energy of the electron drops, upon entering the wall, below the potential step $\chi$ due to conservation of total energy and lateral momentum. It is hence already confined by transmission. Only above the dashed yellow line inelastic backscattering has a chance to bring the electron, once it is inside the wall, back to the interface and, after a successful reverse transmission through the surface potential, back to the plasma. As a result, $S(E, \xi)=\mathcal{T}(E, \xi)$ for $\xi<\sqrt{1-\bar{m}_{e}}$ and $S(E, \xi)<\mathcal{T}(E, \xi)$ for $\xi>\sqrt{1-\bar{m}_{e}}$. This can be more clearly seen in the right panel, where $S(E, \xi)$ (solid curves) and $\mathcal{T}(E, \xi)$ (dashed curves) are plotted as a function of $E$ for representative $\xi$.

The sticking probabilities in Fig. 2 are for $\xi<\xi_{c}$ strongly affected by total reflection caused by the mass mismatch and conservation of energy and lateral momentum. For the latter in-plane homogeneity of the interface is crucial. In reality imperfections may destroy it and lateral momentum may thus not be conserved. To take this possibility into account we include hard core potentials to mimic interfacial scattering centers and adopt the modeling of ballistic electron emission spectroscopy by Smith and coworkers [45] to the calculation of $S$. For a disordered interface we then obtain

$$
\begin{aligned}
\bar{S}(E, \xi) & =\frac{\mathcal{T}(E, \xi)}{1+C / \xi}[1-\overline{\mathcal{E}}(E, \xi)] \\
& +\frac{C / \xi}{1+C / \xi} \int_{\xi_{c}}^{1} d \xi^{\prime} T\left(E, \xi^{\prime}\right)\left[1-\overline{\mathcal{E}}\left(E, \xi^{\prime}\right)\right]
\end{aligned}
$$

where $\overline{\mathcal{E}}(E, \xi)$ is given by (5) with $\mathcal{T}(E, \xi)$ replaced by

$$
\overline{\mathcal{T}}(E, \xi)=\frac{\mathcal{T}(E, \xi)}{1+C / \xi}+\frac{C / \xi}{1+C / \xi} \int_{\xi_{c}}^{1} d \xi^{\prime} \mathcal{T}\left(E, \xi^{\prime}\right)
$$

The parameter $C$ is a fit parameter proportional to the density of scattering centers. It controls the elastic scattering at the interface. In the weak scattering limit, $C \rightarrow 0$ and $\bar{S} \rightarrow S$. We thus recover the sticking probability for the clean interface. In the strong scattering limit, $C \rightarrow \infty$ and we get the sticking probability for the totally disordered interface for which $C$ is irrelevant.

Results for $\mathrm{MgO}$ obtained from Eq. (12) are shown in Fig. 3 for $\xi=1$ (normal incident) and $C=0,1,2$, and $\infty$. We also plot experimental data from electron beam scattering [37]. The agreement between calculated and measured probabilities is astonishing indicating our approach captures essential aspects of electron absorption by the wall at low energies. Dashed curves show $\overline{\mathcal{T}}(E, 1)$, the sticking probability in the absence of internal backscattering. For $C=0, \bar{S}(E, 1)$ deviates strongly from $\overline{\mathcal{T}}(E, 1)$ (black curves), whereas for $C=\infty$ the two quantities approach each other (blue curves). The reason is the angle-averaging at the disordered interface which lessens, for fixed $\xi$, the impact of internal backscattering compared to the knock-out of $\xi$-values by total reflection signalled by the kink at $E=E_{0}$. How close the

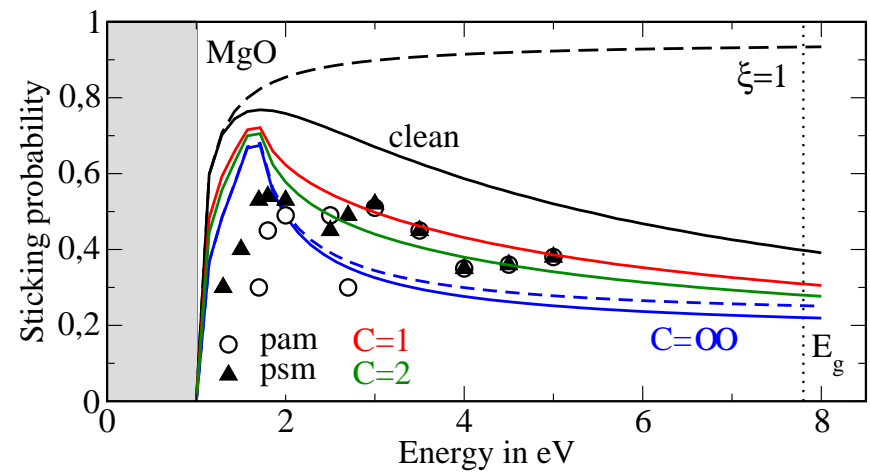

FIG. 3. (color online) Electron sticking probability $\bar{S}(E ; 1)$ for normal incident onto a $\mathrm{MgO}$ wall obtained from Eq. (12) (solid curves). We show data for $C=0$ (black), 1 (red), 2 (green), and $\infty$ (blue) with $\overline{\mathcal{T}}(E ; 1)$ also included for $C=0$ and $C=\infty$ (dashed curves). Symbols are data from electron beam scattering experiments, where two methods, denoted potential adjusted method (pam) and potential subtraction method (psm), have been employed to determine the energy of the electron [37]. Material parameters as in Fig. 2

two curves come to each other, that is, how ineffective internal backscattering becomes depends on $\bar{m}_{e}$ and $\chi$.

To summarize we presented a quantum-mechanical method to calculate the sticking probability $S$ for a lowenergy electron hitting the wall of a bounded plasma. Using the electron's large penetration depth the method expresses $S$ as the probability $\mathcal{T}$ for transmission through the wall's surface potential times the probability $1-\mathcal{E}$ to stay inside it despite of internal backscattering. For $\mathrm{MgO}$ we got in agreement with electron beam scattering data $S<1$, indicating $S \approx 1$, employed in plasma modeling, may not always be justified. Using $S(E, \xi)$ from our approach instead of $S \approx 1$, for instance, in the theory of grain charging in dusty plasmas would reduce the charge of micron-sized $\mathrm{MgO}$ particles by more than ten percent. The method is flexible and numerically efficient. Various scattering processes, including interfacial scattering due to disordered interfaces, can be treated. It could thus guide an experimental effort to develop a realistic description of electron-wall interaction as it occurs in many applications of bounded plasmas.

We acknowledge support by the Deutsche Forschungsgemeinschaft through the Transregional Collaborative Research Center SFB/TRR24.

[1] J. Cazaux, J. Appl. Phys. 111, 064903 (2012).

[2] F. Salvat-Pujol and W. S. M. Werner, Surf. Interface Anal. 45, 873 (2013).

[3] W. S. M. Werner, Surf. Interface Anal. 31, 141 (2001).

[4] A. Klassen, A. Bauereiss, and C. Koerner, J. Phys. D: Appl. Phys. 47, 065307 (2014). 
[5] L. G. Glazov and S. Tougaard, Phys. Rev. B 68, 155409 (2003).

[6] E. Schreiber and H.-J. Fitting, J. Electron Spectros. Relat. Phenom. 124, 25 (2002).

[7] A. Dubus, A. Jablonski, and S. Tougaard, Progr. Surface Science 63, 135 (2000).

[8] M. Vicanek, Surface Science 440, 1 (1999).

[9] S. L. Dudarev, P. Rez, and M. J. Whelan, Phys. Rev. B 51, 3397 (1995).

[10] A. L. Tofterup, Phys. Rev. B 32, 2808 (1985).

[11] K. Kanaya and S. Okayama, J. Phys. D: Appl. Phys. 5, 43 (1972).

[12] R. Dashen, Phys. Rev. 134, A1025 (1964).

[13] M. Bogaczyk, R. Wild, L. Stollenwerk, and H.-E. Wagner, J. Phys. D: Appl. Phys. 45, 465202 (2012).

[14] R. Tschiersch, M. Bogaczyk, and H.-E. Wagner, J. Phys. D: Appl. Phys. 47, 365204 (2014).

[15] F. J. J. Peeters and M. C. M. van de Sanden, Plasma Sources Sci. Technol. 24, 015016 (2015).

[16] P. Tolias, Plasma Phys. Control. Fusion 56, 123002 (2014).

[17] I. Richterová, M. Beránek, J. Pavlů, Z. Němeček, and J. Šáfranková, Phys. Rev. B 81, 075406 (2010).

[18] O. Ishihara, J. Phys. D: Appl. Phys. 40, R121 (2007).

[19] V. E. Fortov, A. V. Ivlev, S. A. Khrapak, A. G. Khrapak, and G. E. Morfill, Phys. Rep. 421, 1 (2005).

[20] D. A. Mendis, Plasma Sources Sci. Technol. 11, A219 (2002).

[21] A. Dunaevsky, Y. Raitses, and N. J. Fisch, Phys. Plasmas 10, 2574 (2003).

[22] S. Barral, K. Makowski, Z. Peradzyński, N. Gascon, and M. Dudeck, Phys. Plasmas 10, 4137 (2003).

[23] M. Lapke, T. Mussenbrock, R. P. Brinkmann, C. Scharwitz, M. Boeke, and J. Winter, Appl. Phys. Lett. 90, 121502 (2007).

[24] M. Shihab, A. T. Elgendy, I. Korolov, A. Derzsi, J. Schulze, D. Eremin, T. Mussenbrock, Z. Donkó, and R. P. Brinkmann, Plasma Sources Sci. Technol. 22, 055013 (2013).

[25] L. Stollenwerk, S. Amiranashvili, J.-P. Boeuf, and H.-G. Purwins, Eur. Phys. J. D 44, 133 (2007).
[26] H. Kersten, H. Deutsch, and G. M. W. Kroesen, Int. J. Mass Spectr. 233, 51 (2004).

[27] R. Brandenburg, V. A. Maiorov, Y. B. Golubovskii, H.E. Wagner, J. Behnke, and J. F. Behnke, J. Phys. D: Appl. Phys. 38, 2187 (2005).

[28] D. Uhrlandt, M. Schmidt, J. F. Behnke, and T. Bindemann, J. Phys. D: Appl. Phys. 33, 2475 (2000).

[29] Y. L. Al'pert, A. V. Gurevich, and L. P. Pitaevskii, Space physics with artificial satellites (Consultants Bureau, New York, 1965).

[30] T. Umebayashi and T. Nakano, Publ. Astron. Soc. Japan 32, 405 (1980).

[31] D. Hollenbach and E. E. Salpeter, J. Chem. Phys. 53, 79 (1970).

[32] F. Bechstedt, Principles of surface physics (Springer Verlag, Berlin, 2003).

[33] R. L. Heinisch, F. X. Bronold, and H. Fehske, Phys. Rev. B 85, 075323 (2012).

[34] T. W. Hickmott, J. Appl. Phys. 36, 1885 (1965).

[35] C. M. Wu and E. S. Yang, Solid-State Electronics 22, 241 (1979).

[36] T. K. Gaylord and K. F. Brennan, J. Appl. Phys. 65, 814 (1989).

[37] C. J. Cook and W. J. Fredericks, J. Chem. Phys 36, 608 (1962).

[38] L. G. Glazov and I. Pázsit, Nucl. Instr. and Meth. B 256, 638 (2007).

[39] B. K. Ridley, Quantum processes in semiconductors (Clarendon Press, Oxford, 1999).

[40] B. K. Ridley, J. Appl. Phys. 84, 4020 (1998).

[41] Y.-S. Kim, S.-H. Yoon, S.-G. Ahn, C.-R. Hong, and H. Yang, Electron. Mater. Lett. 4, 113 (2008).

[42] A. R. Oganov, M. J. Gillan, and G. D. Price, J. Chem. Phys. 118, 10174 (2003).

[43] R. Oswald, E. Kasper, and K. H. Gaukler, J. Electron Spectros. Relat. Phenom. 61, 251 (1993).

[44] Y.-N. Xu and W. Y. Ching, Phys. Rev. B 43, 4461 (1991).

[45] D. L. Smith, E. Y. Lee, and V. Narayanamurti, Phys. Rev. Lett. 80, 2433 (1998). 